\title{
Energy Efficient Spectrum Sensing and Accessing Scheme for Zigbee Cognitive Networks
}

\author{
http://dx.doi.org/10.3991/ijoe.v10i3.3695
}

\author{
F.A. P.Vijayakumar, S.B. Slitta Maria Joseph \\ SRM University, Chennai, India
}

\begin{abstract}
We consider a cognitive radio network that makes access to spectrum licensed to the primary user. In this network, the secondary user will be allowed to use the idle frequency channel of the primary user. It's primarily depending on the proper spectrum sensing. If the channel is seems to be idle the secondary user can occupy the channel but whenever the primary user returns to their frequency channel they have to either switch to the other idle channel or they can wait still on the same channel till it free. In this paper we are considering a cognitive network with one primary user and a secondary user. Secondary user (SU) accessing multiple channels via periodic sensing and spectrum handoff. In our design Implementation is done by utilizing the concept of energy detection algorithm on specific transceiver model of 802.15.4/Zigbee based on Arduino board by analysis of RSSI values of Zigbee devices according to the distance. Also include analyzing of the sensing duration and finding appropriate threshold value for sensing based on Zigbee modems. Energy efficient design is being implemented by utilizing sleeping mode of the Zigbee devices.
\end{abstract}

Index Terms-Arduino, Cognitive Radio, Periodic sensing, RSSI, Switching.

\section{INTRODUCTION}

The Electromagnetic Radio Spectrum, a natural resource, is currently licensed by regulatory bodies for various applications. Presently there is a severe shortage of the spectrum for new applications and systems. Recent studies of Federal communications commission show that $70 \%$ of the channels are occupied in US and also found that 90 percentage of the time licensed frequency bands remain unused [1]. To solve this scenario of the spectrum shortage, the concept of Cognitive radio is implemented. Cognitive radio enables the temporary use of the unused spectrum knows as spectrum hole [2]. While if the secondary user who do not have the license, can have the spectrum while its idle and whenever the primary user returns who have the license, secondary user have to return frequency spectrum to the primary user the moment it returns and either it can wait till primary user again gets free or can go in search of other idle channels. If there will be a delay to return there a collision will occur [3].

Most important thing in this is the channel sensing, it's a critical task. In some cognitive systems, channel sharing is facilitated through periodic sensing [4]. For some system, their energy is critical in that cases it's not suitable to handoff frequently and some time the secondary user choose to wait one the same channel and stop transmission at the cost of increased delay and reduced average throughput [5]. In this paper we propose hardware system with microcontroller, based on Arduino board as to control the spectrum sensing there after the switching of the channel using Zigbee modules in the ISM band and thus to design a system with very less energy consumption.

In the rest of the paper is organized as follows: Section II describes about system model concerning about the transmitter and receiver section and their working mechanism. Section III describes about the implementation of the hardware and software part. Simulation results and discussions are shown in section IV.

\section{SySTEM MODEL}

\section{A. Channel model}

In this section, we will describe the channel model. The primary users are the licensed users who are the one to access the channel same time secondary users are the one, who doesn't have the licensed spectrum and they will be seeking the opportunity to access the channel which is not used by the primary. We assume that there is only one pair of secondary user transmitter and receiver. The secondary user can sense only one channel at a time and access one channel for single transmission [8]. In this paper entire design consist of two parts

1. Monitoring Section

2. Controlling Section

Monitoring section consists of two transceiver which is connected to the PC, can be monitored. Controlling section is fully controlled by the microcontroller. Two transceivers are connected to the microcontroller to one has set as primary user receiver and other as secondary user transmitter. From the receiver get the RSSI value and detecting the idle channel and data transmission on the sensed idle channel.

\section{B. Sensing model}

We consider secondary user as a single channel spectrum sensor. At each interval the secondary user will be checking the presence of the PU. We employ the hypothesis of spectrum sensing by using the energy detection algorithm. In which microcontroller collects all required data from the PU and makes its own decision. Microcontroller makes the final decision according to certain rule and solving a hypothesis testing problem, i.e., the microcontroller determines whether a primary user system is transmitting, given by hypothesis $\mathrm{H}_{1}$, or not, given by hypothesis $\mathrm{H}_{0}[10]$. 


$$
x[n]= \begin{cases}w[n] & \text { under } \mathrm{H}_{0} \\ s[n]+w[n] & \text { under } \mathrm{H}_{1}\end{cases}
$$

Here, $\mathrm{n}=0,1,2 \ldots \mathrm{N}-1, \mathrm{~N}$ represents the index of sample, $w[n]$ specifies the noise and $s[n]$ is the primary signal required to detect. $\mathrm{H}_{0}$ is the hypothesis which means that the received signal consists of the noise only. In case of $\mathrm{H}_{0}$ is true then the decision value will be less than the threshold set by microcontroller. So the controller will be concluding that there is availability of the vacant spectrum. On the other hand, if $\mathrm{H}_{1}$ is true then the received signal has both signal and noise, the decision value will be larger than the threshold. So the microcontroller concludes that the vacant spectrum is not available [6].

\section{EXPERIMENTAL SETUP AND IMPLEMENTATION}

The experimental setup used in this paper is illustrated in Fig. 1 and Fig. 2. We make use of Xbee transceivers which is based on the Zigbee protocol. This low power radio is designed for wireless personal area networks to provide a data rate up to $250 \mathrm{Kbps}$ in indoor/Urban at a range up to $100 \mathrm{~m}$ [7]. Xbee is programmed for 802.15.4 transmission in the ISM $2.4 \mathrm{GHz}$ frequency band. For monitoring part we are using two Xbee as shown in Fig. 1 one is configured as primary user coordinator and other as the secondary user router/end device and these radios are monitored using the software X-CTU provided by the Digi international Inc.

Designed controlling part mainly consists of two Xbee modems and microcontroller. The microcontroller is ATMEL ATMEGA328P based Arduino board called Duemilanove programmed using wiring programming language operating at $16 \mathrm{MHz}$.

Controller has been coded for 1) sensing 2) decision making 3) data transmission. In the controlling part we also have the two Xbee modems configured as primary user router/end device and other one as secondary user coordinator.

\section{A. Monitoring part}

This part mainly consist of the two Xbee modules, they are connected to the personal computer and monitored through the X-CTU software. In this part we will be able to communicate to the Xbees using the transparent /command mode. We make use of AT commands to check current channel used by the Xbee modules in the transmission process and the Table I is the list of all channels that a Xbee make use while it will be in the communication with each other. There is a total of 16 channels can occupied by Xbee in the ISM bands and they can utilize the frequency range of $2.405 \mathrm{GHz}$ to $2.480 \mathrm{GHz}$.

\section{B. Spectrum sensing part}

There has been a lot research on spectrum sensing is going on. As our total design is meant for low power, we consider a simple sensing technique based on energy detection in this paper.

The spectrum sensing part in the microcontroller solves a binary testing problem, choosing a threshold value in a controlled environment observation [9].
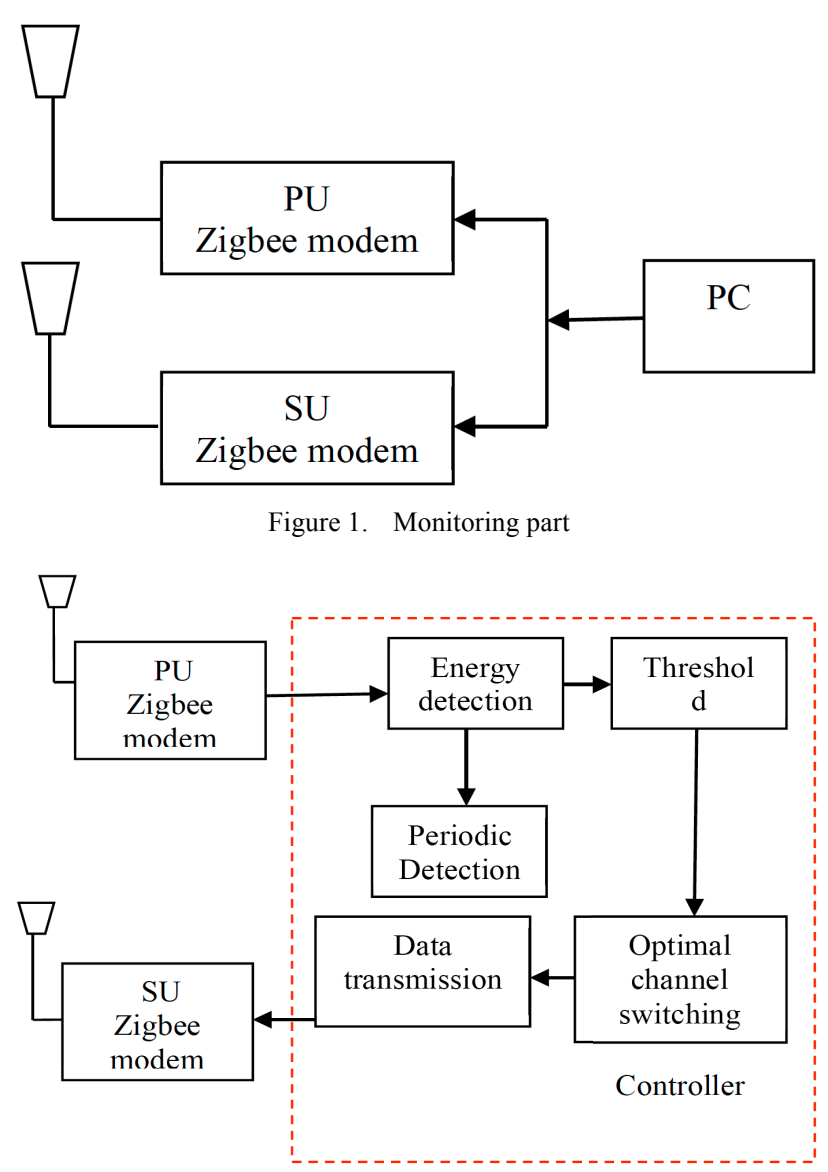

Figure 2. Controlling part

Threshold has been set from the value which is obtained from the received signal strength indication (RSSI) it can obtained from the RSSI pin of the Xbee module or either with help of the AT command. By the value received or sensed from the Xbee is made to compare with threshold value set previously. It has been designed to sense RSSI periodically in interrupt location with a interval of 90 seconds. It's the most critical part in the cognitive radio networks.

\section{Detection and decision making part.}

We can evaluate RSSI values obtained and, next are to make the decision to conclude that the primary user is present or not. If the sensed value is less than the threshold value then primary user is absent and in other case channel not available. In the design it has been coded to detect current channel of the primary user if the channel is available.

\section{Switching and data transmission}

In data transmission section after detection of the available channel the secondary user is allowed to access the channel which is available. The secondary user is allowed to take over the channel used by the primary user and allow the secondary user to change the channel with the help of the AT commands.

While switching to the idle channel the secondary user is allowed to sense whether the primary user returned if so the secondary user have to switch to the another available channel in the ISM $2.4 \mathrm{GHz}$ band. The total process flow is Fig. 3 


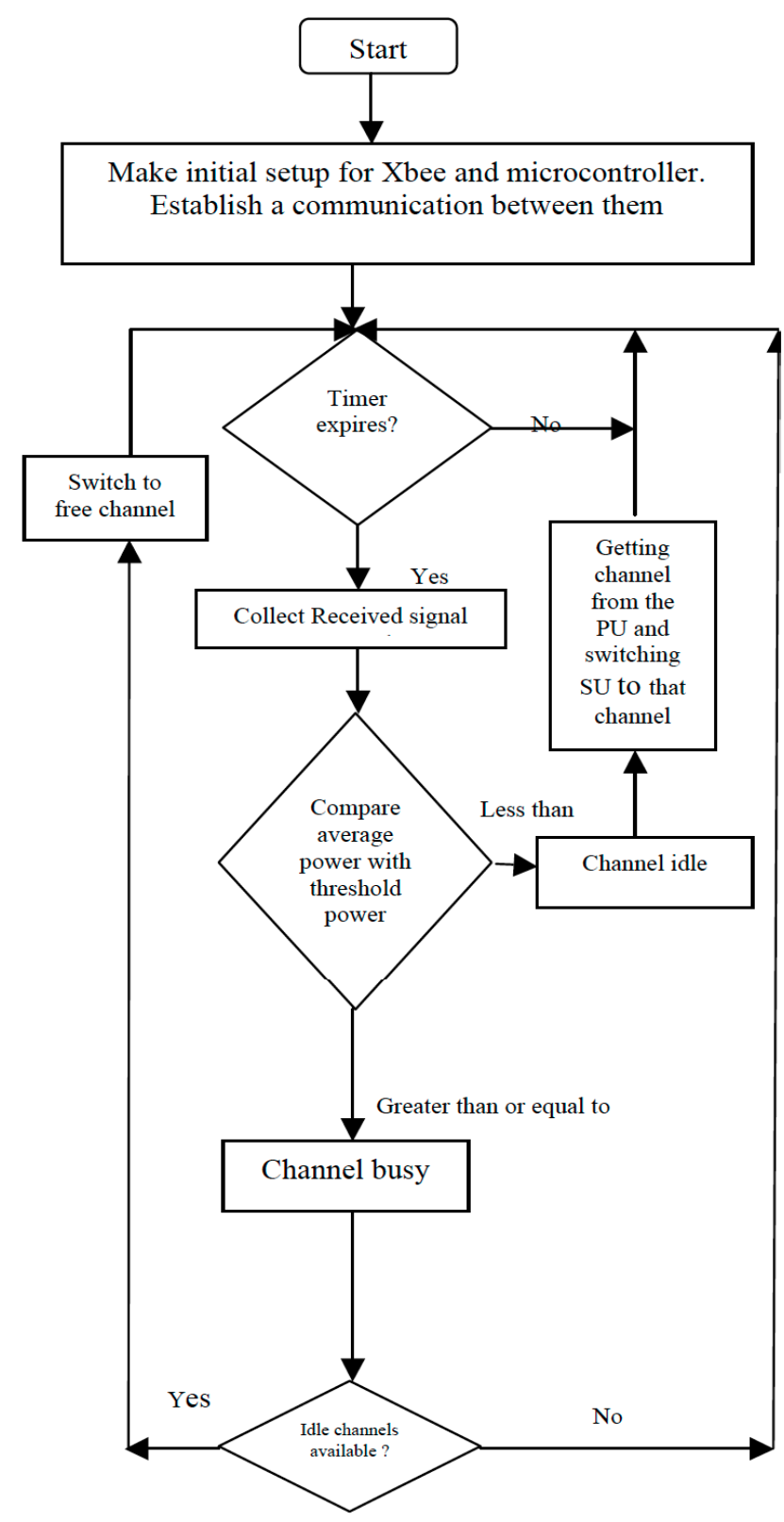

Figure 3. Flow chart of the total system

Programming is done in the Arduino IDE software version 0022 it's an open project written, debugged and supported by Massimo Banzi, David cuartielles, Tom Igoe Gianluca martino and David mellis, Based on processing by Casey Reas and Ben fry.

Microcontroller board having a serial port which is connected to the secondary user coordinator. Primary user has been connected to the soft serial port which is assigned to the $2^{\text {nd }}$ and $3^{\text {rd }}$ pin of the controller board.

\section{Performance Analysis And Results}

In this section, we evaluate the values obtained from the RSSI pin of the Xbee module and the RSSI value obtained from the AT command ATDB. It has been observed that value obtained from the RSSI pin is always above the 600 even though the distance varies. When RSSI value is read from the pin $6^{\text {th }}$ of the Xbee, as a digital output. This uncertainty can be solved be reading the value as the PWM output. Value from the ATDB is variable according to the distance and we can reach a relation between these two.

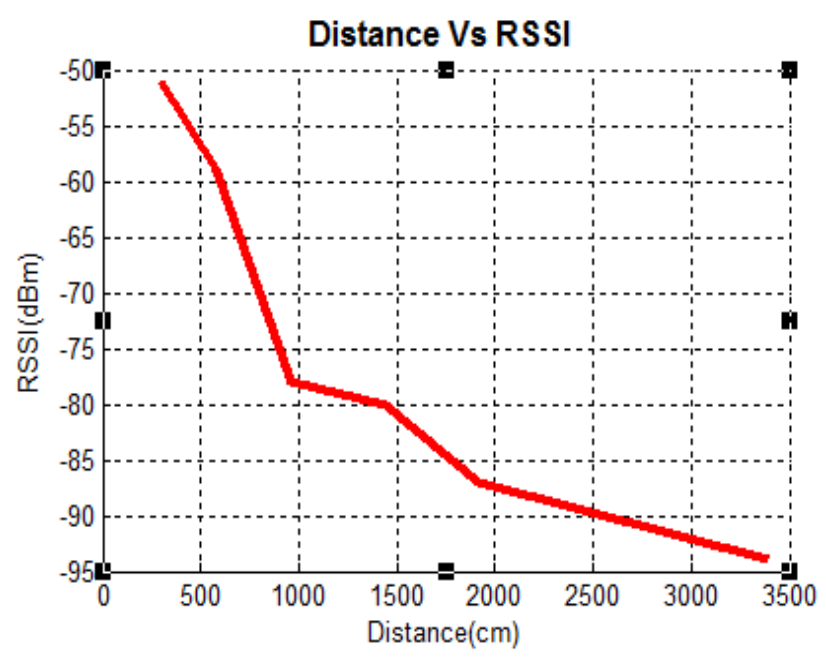

Figure 4. simulation result of relation between distance and RSSI

TABLE I.

CHANNEL DETAILS

\begin{tabular}{|l|c|c|}
\hline $\begin{array}{c}\text { Channel in hexa- } \\
\text { decimal value }\end{array}$ & Frequency (GHz) & SC mask \\
\hline 0X0B & 2.405 & 0x0001 \\
\hline 0X0C & 2.410 & 0X0002 \\
\hline 0X0D & 2.415 & 0X0004 \\
\hline 0X0E & 2.420 & 0X0008 \\
\hline 0X0F & 2.425 & 0X0010 \\
\hline 0X10 & 2.430 & 0X0020 \\
\hline 0X11 & 2.435 & 0X0040 \\
\hline 0X12 & 2.440 & 0X0080 \\
\hline 0X13 & 2.445 & 0X0200 \\
\hline 0X14 & 2.450 & 0X0400 \\
\hline 0X15 & 2.455 & 0X0800 \\
\hline 0X16 & 2.460 & 0X1000 \\
\hline 0X17 & 2.465 & 0X2000 \\
\hline 0X18 & 2.470 & 0X4000 \\
\hline 0X19 & 2.475 & 0X000 \\
\hline 0X1A & 2.480 & \\
\hline
\end{tabular}

In Xbee it will responds in hexadecimal value in the form of $-\mathrm{dBm}$ [6].Range for Xbee indoor we are getting 40 meter without interference it should have a clear path between two Xbee modules then communication can extend few more meters. In channel detection the Xbee almost preferring channel $\mathrm{D}$ in the starting and while switching they need some delay to switch to the other channel. And modules needed a network resetting to follow the channel selected by the coordinator. In detection of the last received packet will be checked within a specified time gap and the value is updated always. And the updated value compared to predefined threshold value. It has been observed that, in switching case the Xbee sometimes not switching to the channel what we described but rather it switching to channels which is more comfortable in the given sixteen channels in the Table.1. As in the observation done using AT command it implies the as the distance varies the RSSI value will get decreased. 


\section{SHORT PAPER}

\section{ENERGy EFFICIENT SPECTRUM SENSING AND ACCESSING SCHEME FOR ZigBEE CogNitive Networks}

\section{FUTURE WORK}

The future applications will be done to reduce the delay in the detection and can carry out for more applications on the including more channels. And the more energy can be saved by enabling the sleep and wake up system in the end devices. When the primary user presence is sensed for a long time it will be possible to make the Xbee modems to sleep for certain amount of time.

\section{REFERENCES}

[1] FCC Spectrum Policy Task Force Fcc report of the spectrum efficiency working group. Nov. 2002.

[2] D. Cabric, S. M. Mishra, And R. W. Brodersen, "Implementation Issues In Spectrum Sensing For Cognitive Radios", In Proc. 38th Asilomar Conference On Signals, Systems And Computers, Pp. 772776, Nov. 2004.

[3] C.-W. Wang, L.-C.Wang, and F. Adachi, "Modeling and analysis for reactive decision spectrum handoff in cognitive radio networks," in Proc. IEEE Globecom, Dec 2010, pp. 1-6.

[4] Y.-C. Liang, Y. Zeng, E. C. Peh, and A. T. Hoang, "Sensingthroughput tradeoff for cognitive radio networks," IEEE Trans. Wireless Commun., vol. 7, no. 4, pp. 1326-1337, Apr. 2008. http://dx.doi.org/10.1109/TWC.2008.060869

[5] S. Maleki, A. Pandharipande, and G. Leus, "Energy-efficient distributedspectrum sensing for cogntive sensor netowrks," IEEE Sens. J., vol. 11,no. 3, pp. 565-573, Mar. 2011. http://dx.doi.org/10.1109/JSEN.2010.2051327
[6] Dynamic spectrum acess and management in Cognitive Radio Networks-Hossain, Niyato and Han published by Cambridge University Press.

[7] xbee product manual" $\mathrm{XBee}^{\mathrm{TM}} \mathrm{ZNet} 2.5 / \mathrm{XBee}^{\mathrm{P} R O^{\mathrm{TM}}} \mathrm{ZNet} 2.5$ OEM RF Modules"

[8] He li, Xinxin Feng, Xiaoying Gan, Zhongren cao. "Joint spectrum sensing and transmission strategy for energy-efficient cognitive radio networks" $20138^{\text {th }}$ international conference on cognitive radio oriented wireless networks.

[9] Sina maleki, Ashish Pandharipande, Geert leus. “ Energy-efficient distributed spectrum sensing for cognitive sensor networks " IEEE sensors journal, VOL. 11, No.3, March 2011.

[10] Sundeep Prabhakar chepuri, Ruden de Franscisco and Geert Leus. "Performance evaluation of an IEEE 802.15.4 cognitive radio link in the 2360-2400 MHz band " IEEE WCNC 2011.

\section{AUTHORS}

F. A. P.Vijayakumar is with the SRM University as Assistant professor with the Department of Electronics and communication. (vijayakumar.p@ktr.srmuniv.ac.in)

S. B. Slitta Maria Joseph is with SRM University, Chennai, India. She is now with the Department of Electronics and communication. (slittacjosephr@gmail.com).

Submitted 21 March 2014. Published as re-submitted by the authors 28 April 2014. 